\title{
EXISTENCE RESULTS FOR FRACTIONAL DIFFERENTIAL INCLUSIONS WITH SEPARATED BOUNDARY CONDITIONS
}

\author{
BASHIR AHMAD
}

\begin{abstract}
In this paper, we apply Bohnenblust-Karlins fixed point theorem to prove the existence of solutions for a class of fractional differential inclusions with separated boundary conditions. Some applications of the main result are also presented.
\end{abstract}

\section{Introduction}

Fractional-order models are found to be more adequate than integer-order models in some real world problems as fractional derivatives provide an excellent tool for the description of memory and hereditary properties of various materials and processes. The mathematical modeling of systems and processes in the fields of physics, chemistry, aerodynamics, electro dynamics of complex medium, polymer rheology, etc. involves derivatives of fractional order. In consequence, the subject of fractional differential equations is gaining much importance and attention. For details and examples, see [1-4, 10, 12, 15-16, 18, 22-24, 26] and the references therein.

Differential inclusions arise in the mathematical modeling of certain problems in economics, optimal control, etc. and are widely studied by many authors, see $[7,20,25]$ and the references therein. For some recent development on differential inclusions, we refer the reader to the references $[8-9,13,19,21]$.

Chang and Nieto [7] discussed the existence of solutions for the fractional boundary value problem:

$$
\left\{\begin{array}{c}
{ }_{0}^{c} D_{t}^{\delta} y(t) \in F(t, y(t)), t \in[0,1], \delta \in(1,2) \\
y(0)=\alpha, y(1)=\beta, \alpha, \beta \neq 0 .
\end{array}\right.
$$

In this paper, we consider the following fractional differential inclusions with separated boundary conditions

$$
\left\{\begin{array}{l}
{ }^{c} D^{q} x(t) \in F(t, x(t)), t \in[0,1], 1<q \leq 2 \\
\alpha x(0)+\beta x^{\prime}(0)=\gamma_{1}, \alpha x(1)+\beta x^{\prime}(1)=\gamma_{2}
\end{array}\right.
$$

Received February 27, 2009; Revised April 24, 2009.

2000 Mathematics Subject Classification. 34A12, 34A40.

Key words and phrases. fractional differential inclusions, separated boundary conditions, Bohnenblust-Karlins fixed point theorem. 
where ${ }^{c} D^{q}$ denote the Caputo fractional derivative of order $q, F:[0,1] \times \mathbb{R} \rightarrow$ $2^{\mathbb{R}} \backslash\{\emptyset\}$, and $\alpha>0, \beta \geq 0, \gamma_{1}, \gamma_{2}$ are real numbers. Bohnenblust-Karlin fixed point theorem is applied to prove the existence of solutions of (1.1).

\section{Preliminaries}

Let $C([0,1])$ denote a Banach space of continuous functions from $[0,1]$ into $\mathbb{R}$ with the norm $\|x\|=\sup _{t \in[0,1]}\{|x(t)|\}$. Let $L^{1}([0,1], \mathbb{R})$ be the Banach space of functions $x:[0,1] \rightarrow \mathbb{R}$ which are Lebesgue integrable and normed by $\|x\|_{L^{1}}=\int_{0}^{1}|x(t)| d t$.

Now we recall some basic definitions on multi-valued maps $[11,14]$.

Let $(X,\|\cdot\|)$ be a Banach space. Then a multi-valued map $G: X \rightarrow 2^{X}$ is convex (closed) valued if $G(x)$ is convex (closed) for all $x \in X$. The map $G$ is bounded on bounded sets if $G(\mathbb{B})=\cup_{x \in \mathbb{B}} G(x)$ is bounded in $X$ for any bounded set $\mathbb{B}$ of $X$ (i.e., $\left.\sup _{x \in \mathbb{B}}\{\sup \{|y|: y \in G(x)\}\}<\infty\right)$ ). $G$ is called upper semi-continuous (u.s.c.) on $X$ if for each $x_{0} \in X$, the set $G\left(x_{0}\right)$ is a nonempty closed subset of $X$, and if for each open set $\mathbb{B}$ of $X$ containing $G\left(x_{0}\right)$, there exists an open neighborhood $\mathcal{N}$ of $x_{0}$ such that $G(\mathcal{N}) \subseteq \mathbb{B}$. $G$ is said to be completely continuous if $G(\mathbb{B})$ is relatively compact for every bounded subset $\mathbb{B}$ of $X$. If the multi-valued map $G$ is completely continuous with nonempty compact values, then $G$ is u.s.c. if and only if $G$ has a closed graph, i.e., $x_{n} \rightarrow x_{*}, y_{n} \rightarrow y_{*}, y_{n} \in G\left(x_{n}\right)$ imply $y_{*} \in G\left(x_{*}\right)$. In the following study, $B C C(X)$ denotes the set of all nonempty bounded, closed and convex subset of $X$. $G$ has a fixed point if there is $x \in X$ such that $x \in G(x)$.

Let us record some definitions on fractional calculus [15, 22, 24].

Definition 2.1. For a function $g:[0, \infty) \rightarrow \mathbb{R}$, the Caputo derivative of fractional order $q$ is defined as

$$
{ }^{c} D^{q} g(t)=\frac{1}{\Gamma(n-q)} \int_{0}^{t}(t-s)^{n-q-1} g^{(n)}(s) d s, n-1<q \leq n, q>0,
$$

where $\Gamma$ denotes the gamma function.

Definition 2.2. The Riemann-Liouville fractional integral of order $q$ for a function $g$ is defined as

$$
I^{q} g(t)=\frac{1}{\Gamma(q)} \int_{0}^{t} \frac{g(s)}{(t-s)^{1-q}} d s, q>0
$$

provided the right hand side is pointwise defined on $(0, \infty)$.

Definition 2.3. The Riemann-Liouville fractional derivative of order $q$ for a function $g$ is defined by

$$
D^{q} g(t)=\frac{1}{\Gamma(n-q)}\left(\frac{d}{d t}\right)^{n} \int_{0}^{t} \frac{g(s)}{(t-s)^{q-n+1}} d s, n-1<q \leq n, q>0,
$$

provided the right hand side is pointwise defined on $(0, \infty)$. 
Here, we remark that the Caputo derivative becomes the conventional $n$ th derivative of the function as $q \rightarrow n$ and the initial/boundary conditions for fractional differential equations retain the same form as that of ordinary differential equations with integer order derivatives. On the other hand, the Riemann-Liouville fractional derivative could hardly produce the physical interpretation of the initial/boundary conditions required for the initial/boundary value problems involving fractional differential equations. Moreover, the Caputo derivative for a constant is zero while the Riemann-Liouville fractional derivative of a constant is nonzero. For more details, see [24].

For the forthcoming analysis, we need the following assumptions:

$\left(\mathbf{A}_{1}\right)$ Let $F:[0,1] \times \mathbb{R} \rightarrow B C C(\mathbb{R}) ;(t, x) \rightarrow F(t, x)$ be measurable with respect to $t$ for each $x \in \mathbb{R}$, u.s.c. with respect to $x$ for a.e. $t \in[0,1]$, and for each fixed $x \in \mathbb{R}$, the set $S_{F, y}:=\left\{f \in L^{1}([0,1], \mathbb{R}): f(t) \in\right.$ $F(t, x)$ for a.e. $t \in[0,1]\}$ is nonempty.

$\left(\mathbf{A}_{2}\right)$ For each $r>0$, there exists a function $m_{r} \in L^{1}\left([0,1], \mathbb{R}_{+}\right)$such that $\|F(t, x)\|=\sup \{|v|: v(t) \in F(t, x)\} \leq m_{r}(t)$ for each $(t, x) \in[0,1] \times \mathbb{R}$ with $|x| \leq r$, and

$$
\liminf _{r \rightarrow+\infty}\left(\frac{\int_{0}^{1} m_{r}(t) d t}{r}\right)=\omega<\infty .
$$

In relation to $(1.1)$, we define

$$
\Lambda=\left(\frac{2 \alpha+\beta}{\alpha \Gamma(q)}+\frac{\beta^{2}+\alpha \beta}{\alpha^{2} \Gamma(q-1)}\right)^{-1} .
$$

As argued in [3], a function $x \in C([0,1])$ is a solution of the problem (1.1) if there exists a function $f \in L^{1}([0,1], \mathbb{R})$ such that $f(t) \in F(t, x)$ a.e. on $[0,1]$ and

$$
x(t)=\int_{0}^{1} G(t, s) f(s) d s+\frac{1}{\alpha^{2}}\left[(\alpha(1-t)+\beta) \gamma_{1}+(\alpha t-\beta) \gamma_{2}\right],
$$

where $G(t, s)$ is the Green's function given by

$$
G(t, s)=\left\{\begin{array}{c}
\frac{\alpha(t-s)^{q-1}+(\beta-\alpha t)(1-s)^{q-1}}{\alpha \Gamma(q)}+\frac{\beta(\beta-\alpha t)(1-s)^{q-2}}{\alpha^{2} \Gamma(q-1)}, s \leq t, \\
\frac{(\beta-\alpha t)(1-s)^{q-1}}{\alpha \Gamma(q)}+\frac{\beta(\beta-\alpha t)(1-s)^{q-2}}{\alpha^{2} \Gamma(q-1)}, t \leq s .
\end{array}\right.
$$

Now we state the following lemmas which are necessary to establish the main result.

Lemma 2.1 (Bohnenblust-Karlin [5]). Let D be a nonempty subset of a Banach space $X$, which is bounded, closed, and convex. Suppose that $G: D \rightarrow 2^{X} \backslash\{0\}$ is u.s.c. with closed, convex values such that $G(D) \subset D$ and $\overline{G(D)}$ is compact. Then $G$ has a fixed point.

Lemma 2.2 ([17]). Let I be a compact real interval. Let $F$ be a multi-valued map satisfying $\left(A_{1}\right)$ and let $\Theta$ be linear continuous from $L^{1}(I, \mathbb{R}) \rightarrow C(I)$. Then 
the operator $\Theta \circ S_{F}: C(I) \rightarrow B C C(C(I)), x \mapsto\left(\Theta \circ S_{F}\right)(x)=\Theta\left(S_{F, x}\right)$ is a closed graph operator in $C(I) \times C(I)$.

\section{Main result}

Theorem 3.1. Suppose that the assumptions $\left(A_{1}\right)$ and $\left(A_{2}\right)$ are satisfied, and

$$
\omega<\Lambda \text {, }
$$

where $\omega$ and $\Lambda$ are respectively given by (2.1) and (2.2). Then the boundary value problem (1.1) has at least one solution on $[0,1]$.

Proof. To transform the problem (1.1) into a fixed point problem, we define a multi-valued map $\Omega: C([0,1]) \rightarrow 2^{C([0,1])}$ as

$$
\begin{aligned}
\Omega(x)=\{ & h \in C([0,1]): h(t)=\frac{1}{\Gamma(q)} \int_{0}^{t}(t-s)^{q-1} f(s) d s \\
& +\int_{0}^{1}\left(\frac{(\beta-\alpha t)(1-s)^{q-1}}{\alpha \Gamma(q)}+\frac{\beta(\beta-\alpha t)(1-s)^{q-2}}{\alpha^{2} \Gamma(q-1)}\right) f(s) d s \\
& \left.+\frac{1}{\alpha^{2}}\left[(\alpha(1-t)+\beta) \gamma_{1}+(\alpha t-\beta) \gamma_{2}\right], f \in S_{F, x}\right\} .
\end{aligned}
$$

Now we prove that $\Omega$ satisfies all the assumptions of Lemma 2.1, and thus $\Omega$ has a fixed point which is a solution of the problem (1.1). As a first step, we show that $\Omega(x)$ is convex for each $x \in C([0,1])$. For that, let $h_{1}, h_{2} \in \Omega(x)$. Then there exist $f_{1}, f_{2} \in S_{F, x}$ such that for each $t \in[0,1]$, we have

$$
\begin{aligned}
h_{i}(t)= & \frac{1}{\Gamma(q)} \int_{0}^{t}(t-s)^{q-1} f_{i}(s) d s \\
& +\int_{0}^{1}\left(\frac{(\beta-\alpha t)(1-s)^{q-1}}{\alpha \Gamma(q)}+\frac{\beta(\beta-\alpha t)(1-s)^{q-2}}{\alpha^{2} \Gamma(q-1)}\right) f_{i}(s) d s \\
& +\frac{1}{\alpha^{2}}\left[(\alpha(1-t)+\beta) \gamma_{1}+(\alpha t-\beta) \gamma_{2}\right], i=1,2 .
\end{aligned}
$$

Let $0 \leq \lambda \leq 1$. Then, for each $t \in[0,1]$, we have

$$
\begin{aligned}
& {\left[\lambda h_{1}+(1-\lambda) h_{2}\right](t) } \\
= & \frac{1}{\Gamma(q)} \int_{0}^{t}(t-s)^{q-1}\left[\lambda f_{1}(s)+(1-\lambda) f_{2}(s)\right] d s \\
& +\int_{0}^{1}\left(\frac{(\beta-\alpha t)(1-s)^{q-1}}{\alpha \Gamma(q)}+\frac{\beta(\beta-\alpha t)(1-s)^{q-2}}{\alpha^{2} \Gamma(q-1)}\right)\left[\lambda f_{1}(s)+(1-\lambda) f_{2}(s)\right] d s \\
& +\frac{1}{\alpha^{2}}\left[(\alpha(1-t)+\beta) \gamma_{1}+(\alpha t-\beta) \gamma_{2}\right] .
\end{aligned}
$$

Since $S_{F, x}$ is convex ( $F$ has convex values), therefore it follows that $\lambda h_{1}+(1-$ $\lambda) h_{2} \in \Omega(x)$.

Next we show that there exists a positive number $r$ such that $\Omega\left(B_{r}\right) \subseteq B_{r}$, where $B_{r}=\{x \in C([0,1]):\|x\| \leq r\}$. Clearly $B_{r}$ is a bounded closed convex set 
in $C([0,1])$ for each positive constant $r$. If it is not true, then for each positive number $r$, there exists a function $x_{r} \in B_{r}, h_{r} \in \Omega\left(x_{r}\right)$ with $\left\|\Omega\left(x_{r}\right)\right\|>r$, and

$$
\begin{aligned}
h_{r}(t)= & \frac{1}{\Gamma(q)} \int_{0}^{t}(t-s)^{q-1} f_{r}(s) d s \\
& +\int_{0}^{1}\left(\frac{(\beta-\alpha t)(1-s)^{q-1}}{\alpha \Gamma(q)}+\frac{\beta(\beta-\alpha t)(1-s)^{q-2}}{\alpha^{2} \Gamma(q-1)}\right) f_{r}(s) d s \\
& +\frac{1}{\alpha^{2}}\left[(\alpha(1-t)+\beta) \gamma_{1}+(\alpha t-\beta) \gamma_{2}\right] \text { for some } f_{r} \in S_{F, x_{r}} .
\end{aligned}
$$

On the other hand, in view of $\left(A_{2}\right)$, we have

$$
\begin{aligned}
r< & \left\|\Omega\left(x_{r}\right)\right\| \\
\leq & \frac{1}{\Gamma(q)} \int_{0}^{t}|t-s|^{q-1}\left|f_{r}(s)\right| d s \\
& +\int_{0}^{1}\left|\frac{(\beta-\alpha t)(1-s)^{q-1}}{\alpha \Gamma(q)}+\frac{\beta(\beta-\alpha t)(1-s)^{q-2}}{\alpha^{2} \Gamma(q-1)}\right|\left|f_{r}(s)\right| d s \\
& +\frac{1}{\alpha^{2}}\left|(\alpha(1-t)+\beta) \gamma_{1}+(\alpha t-\beta) \gamma_{2}\right| \\
\leq & \left(\frac{2 \alpha+\beta}{\alpha \Gamma(q)}+\frac{\beta^{2}+\alpha \beta}{\alpha^{2} \Gamma(q-1)}\right) \int_{0}^{1} m_{r}(s) d s+\frac{\alpha+\beta}{\alpha^{2}}\left(\left|\gamma_{1}\right|+\left|\gamma_{2}\right|\right) .
\end{aligned}
$$

Dividing both sides by $r$ and taking the lower limit as $r \rightarrow \infty$, we find that

$$
\omega \geq\left(\frac{2 \alpha+\beta}{\alpha \Gamma(q)}+\frac{\beta^{2}+\alpha \beta}{\alpha^{2} \Gamma(q-1)}\right)^{-1}=\Lambda,
$$

which contradicts (3.1). Hence there exists a positive number $r^{\prime}$ such that $\Omega\left(B r^{\prime}\right) \subseteq B r^{\prime}$

Now we show that $\Omega\left(B_{r^{\prime}}\right)$ is equi-continuous. Let $t^{\prime}, t^{\prime \prime} \in[0,1]$ with $t^{\prime}<t^{\prime \prime}$. Let $x \in B_{r^{\prime}}$ and $h \in \Omega(x)$. Then there exists $f \in S_{F, x}$ such that for each $t \in[0,1]$, we have

$$
\begin{aligned}
h(t)= & \frac{1}{\Gamma(q)} \int_{0}^{t}(t-s)^{q-1} f(s) d s \\
& +\int_{0}^{1}\left(\frac{(\beta-\alpha t)(1-s)^{q-1}}{\alpha \Gamma(q)}+\frac{\beta(\beta-\alpha t)(1-s)^{q-2}}{\alpha^{2} \Gamma(q-1)}\right) f(s) d s \\
& +\frac{1}{\alpha^{2}}\left[(\alpha(1-t)+\beta) \gamma_{1}+(\alpha t-\beta) \gamma_{2}\right] .
\end{aligned}
$$

Using (3.2), we obtain

$$
\begin{aligned}
& \left|h\left(t^{\prime \prime}\right)-h\left(t^{\prime}\right)\right| \\
= & \mid \frac{1}{\Gamma(q)} \int_{0}^{t^{\prime \prime}}\left(t^{\prime \prime}-s\right)^{q-1} f(s) d s
\end{aligned}
$$




$$
\begin{aligned}
& +\int_{0}^{1}\left(\frac{\left(\beta-\alpha t^{\prime \prime}\right)(1-s)^{q-1}}{\alpha \Gamma(q)}+\frac{\beta\left(\beta-\alpha t^{\prime \prime}\right)(1-s)^{q-2}}{\alpha^{2} \Gamma(q-1)}\right) f(s) d s \\
& +\frac{1}{\alpha^{2}}\left[\left(\alpha\left(1-t^{\prime \prime}\right)+\beta\right) \gamma_{1}+\left(\alpha t^{\prime \prime}-\beta\right) \gamma_{2}\right]-\frac{1}{\Gamma(q)} \int_{0}^{t^{\prime}}\left(t^{\prime}-s\right)^{q-1} f(s) d s \\
& -\int_{0}^{1}\left(\frac{\left(\beta-\alpha t^{\prime}\right)(1-s)^{q-1}}{\alpha \Gamma(q)}+\frac{\beta\left(\beta-\alpha t^{\prime}\right)(1-s)^{q-2}}{\alpha^{2} \Gamma(q-1)}\right) f(s) d s \\
& -\frac{1}{\alpha^{2}}\left[\left(\alpha\left(1-t^{\prime}\right)+\beta\right) \gamma_{1}+\left(\alpha t^{\prime}-\beta\right) \gamma_{2}\right] \mid \\
\leq & \int_{0}^{t^{\prime}}\left(\frac{\left(t^{\prime \prime}-s\right)^{q-1}-\left(t^{\prime}-s\right)^{q-1}}{\Gamma(q)}\right) f(s) d s|+| \int_{t^{\prime}}^{t^{\prime \prime}} \frac{\left(t^{\prime \prime}-s\right)^{q-1}}{\Gamma(q)} f(s) d s \mid \\
& +\left|-\left(t^{\prime \prime}-t^{\prime}\right) \int_{0}^{1}\left[\frac{(1-s)^{q-1}}{\Gamma(q)}+\frac{\beta(1-s)^{q-2}}{\alpha \Gamma(q-1)}\right] f(s) d s\right| \\
& +\frac{1}{\alpha}\left|\left(t^{\prime \prime}-t^{\prime}\right)\left(\gamma_{2}-\gamma_{1}\right)\right| \\
\leq & \frac{1}{\Gamma(q)} \int_{0}^{t^{\prime}}\left|\left(t^{\prime \prime}-s\right)^{q-1}-\left(t^{\prime}-s\right)^{q-1}\right| m_{r^{\prime}}(s) d s+\frac{1}{\Gamma(q)} \int_{t^{\prime}}^{t^{\prime \prime}} m_{r^{\prime}}(s) d s \\
& +\left(t^{\prime \prime}-t^{\prime}\right)\left[\left(\frac{1}{\Gamma(q)}+\frac{\beta}{\alpha \Gamma(q-1)}\right) \int_{0}^{1} m_{r^{\prime}}(s) d s+\frac{1}{\alpha}\left|\left(\gamma_{2}-\gamma_{1}\right)\right|\right] .
\end{aligned}
$$

Obviously the right hand side of the above inequality tends to zero independently of $x \in B_{r^{\prime}}$ as $t^{\prime \prime} \rightarrow t^{\prime}$. Thus, $\Omega$ is equi-continuous. As $\Omega$ satisfies the above three assumptions, therefore it follows by Ascoli-Arzela theorem that $\Omega$ is a compact multi-valued map.

Finally, we show that $\Omega$ has a closed graph. Let $x_{n} \rightarrow x_{*}, h_{n} \in \Omega\left(x_{n}\right)$ and $h_{n} \rightarrow h_{*}$. We will show that $h_{*} \in \Omega\left(x_{*}\right)$. By the relation $h_{n} \in \Omega\left(x_{n}\right)$, we mean that there exists $f_{n} \in S_{F, x_{n}}$ such that for each $t \in[0,1]$,

$$
\begin{aligned}
h_{n}(t)= & \frac{1}{\Gamma(q)} \int_{0}^{t}(t-s)^{q-1} f_{n}(s) d s \\
& +\int_{0}^{1}\left(\frac{(\beta-\alpha t)(1-s)^{q-1}}{\alpha \Gamma(q)}+\frac{\beta(\beta-\alpha t)(1-s)^{q-2}}{\alpha^{2} \Gamma(q-1)}\right) f_{n}(s) d s \\
& +\frac{1}{\alpha^{2}}\left[(\alpha(1-t)+\beta) \gamma_{1}+(\alpha t-\beta) \gamma_{2}\right] .
\end{aligned}
$$

Thus we need to show that there exists $f_{*} \in S_{F, x_{*}}$ such that for each $t \in[0,1]$,

$$
\begin{aligned}
h_{*}(t)= & \frac{1}{\Gamma(q)} \int_{0}^{t}(t-s)^{q-1} f_{*}(s) d s \\
& +\int_{0}^{1}\left(\frac{(\beta-\alpha t)(1-s)^{q-1}}{\alpha \Gamma(q)}+\frac{\beta(\beta-\alpha t)(1-s)^{q-2}}{\alpha^{2} \Gamma(q-1)}\right) f_{*}(s) d s \\
& +\frac{1}{\alpha^{2}}\left[(\alpha(1-t)+\beta) \gamma_{1}+(\alpha t-\beta) \gamma_{2}\right] .
\end{aligned}
$$


Let us consider the continuous linear operator $\Theta: L^{1}([0,1], \mathbb{R}) \rightarrow C([0,1])$ so that

$$
\begin{aligned}
f \mapsto \Theta(f)(t)= & \frac{1}{\Gamma(q)} \int_{0}^{t}(t-s)^{q-1} f(s) d s \\
& +\int_{0}^{1}\left(\frac{(\beta-\alpha t)(1-s)^{q-1}}{\alpha \Gamma(q)}+\frac{\beta(\beta-\alpha t)(1-s)^{q-2}}{\alpha^{2} \Gamma(q-1)}\right) f(s) d s \\
& +\frac{1}{\alpha^{2}}\left[(\alpha(1-t)+\beta) \gamma_{1}+(\alpha t-\beta) \gamma_{2}\right] .
\end{aligned}
$$

Observe that

$$
\begin{aligned}
& \left\|h_{n}(t)-h_{*}(t)\right\| \\
= & \| \frac{1}{\Gamma(q)} \int_{0}^{t}(t-s)^{q-1}\left(f_{n}(s)-f_{*}(s)\right) d s \\
& +\int_{0}^{1}\left(\frac{(\beta-\alpha t)(1-s)^{q-1}}{\alpha \Gamma(q)}+\frac{\beta(\beta-\alpha t)(1-s)^{q-2}}{\alpha^{2} \Gamma(q-1)}\right)\left(f_{n}(s)-f_{*}(s)\right) d s \| \\
\rightarrow & 0 \quad \text { as } n \rightarrow \infty .
\end{aligned}
$$

Thus, it follows by Lemma 2.2 that $\Theta \circ S_{F}$ is a closed graph operator. Further, we have $h_{n}(t) \in \Theta\left(S_{F, x_{n}}\right)$. Since $x_{n} \rightarrow x_{*}$, therefore, Lemma 2.2 yields

$$
\begin{aligned}
h_{*}(t)= & \frac{1}{\Gamma(q)} \int_{0}^{t}(t-s)^{q-1} f_{*}(s) d s \\
& +\int_{0}^{1}\left(\frac{(\beta-\alpha t)(1-s)^{q-1}}{\alpha \Gamma(q)}+\frac{\beta(\beta-\alpha t)(1-s)^{q-2}}{\alpha^{2} \Gamma(q-1)}\right) f_{*}(s) d s \\
& +\frac{1}{\alpha^{2}}\left[(\alpha(1-t)+\beta) \gamma_{1}+(\alpha t-\beta) \gamma_{2}\right] .
\end{aligned}
$$

Hence, we conclude that $\Omega$ is a compact multi-valued map, u.s.c. with convex closed values. Thus, all the assumptions of Lemma 2.1 are satisfied and so by the conclusion of Lemma 2.1, $\Omega$ has a fixed point $x$ which is a solution of the problem (1.1). This completes the proof.

Remark 3.1. If we take $F(t, x)=\{f(t, x)\}$, where $f:[0,1] \times \mathbb{R} \rightarrow \mathbb{R}$ is a continuous function, then our results correspond to a single-valued problem (a new result).

Remark 3.2. The results of reference [7] appear as a special case of the results of this paper if we choose $\alpha=1, \beta=0$ in (1.1).

Applications. As an application of Theorem 3.1, we discuss two cases in relation to the nonlinearity $F$ in (1.1), namely, $F$ has (a) sub-linear growth in its second variable (b) linear growth in its second variable (state variable). In case of sub-linear growth, there exist functions $\eta(t), \rho(t) \in L^{1}\left([0,1], \mathbb{R}_{+}\right), \mu \in$ $[0,1)$ such that $\|F(t, x)\| \leq \eta(t)|x|^{\mu}+\rho(t)$ for each $(t, x) \in[0,1] \times \mathbb{R}$. In this case, $m_{r}(t)=\eta(t) r^{\mu}+\rho(t)$ and the condition (3.1) is $0<\Lambda$. For the linear 
growth, the nonlinearity $F$ satisfies the relation $\|F(t, x)\| \leq \eta(t)|x|+\rho(t)$ for each $(t, x) \in[0,1] \times \mathbb{R}$. In this case $m_{r}(t)=\eta(t) r+\rho(t)$ and the condition (3.1) becomes $\|\eta\|_{L^{1}}<\lambda$. In both the cases, the boundary value problem (1.1) has at least one solution on $[0,1]$.

Examples. (a) We consider $F(t, x)$ in (1.1) satisfying the condition

$$
\|F(t, x)\| \leq \eta(t)|x|^{1 / 3}+\rho(t),
$$

where, $\eta(t), \rho(t) \in L^{1}\left([0,1], \mathbb{R}_{+}\right)$. In this case, we find that the conclusion of Theorem 3.1 applies with the condition (3.1) taking the form $0<\Lambda$.

(b) Let us take $F(t, x)$ such that $\|F(t, x)\| \leq \frac{1}{(1+t)^{2}}|x|+e^{-t}$ in (1.1). In this case, (3.1) takes the form $\frac{1}{2}<\Lambda$. Thus, by Theorem 3.1, the problem (1.1) has at least one solution on $[0,1]$.

Acknowledgement. The author thanks the anonymous referee for a careful reading of the manuscript.

\section{References}

[1] B. Ahmad and J. J. Nieto, Existence results for nonlinear boundary value problems of fractional integrodifferential equations with integral boundary conditions, Bound. Value Probl. 2009, Art. ID 708576, 11 pp.

[2] __ Existence results for a coupled system of nonlinear fractional differential equations with three-point boundary conditions, Comput. Math. Appl. 58 (2009), no. 9, 1838-1843.

[3] _ Existence results for nonlinear impulsive hybrid boundary value problems involving fractional differential equations, Nonlinear Anal. Hybrid Syst. 3 (2009), no. 3, 251-258.

[4] B. Ahmad and S. Sivasundaram, Existence and uniqueness results for nonlinear boundary value problems of fractional differential equations with separated boundary conditions, Commun. Appl. Anal. 13 (2009), no. 1, 121-127.

[5] H. F. Bohnenblust and S. Karlin, On a theorem of Ville, Contributions to the Theory of Games, 155-160. Annals of Mathematics Studies, no. 24. Princeton University Press, Princeton, N. J., 1950.

[6] Y.-K. Chang, W. T. Li, and J. J. Nieto, Controllability of evolution differential inclusions in Banach spaces, Nonlinear Anal. 67 (2007), no. 2, 623-632.

[7] Y.-K. Chang and J. J. Nieto, Some new existence results for fractional differential inclusions with boundary conditions, Math. Comput. Modelling 49 (2009), no. 3-4, 605609.

[8] _ Existence of solutions for impulsive neutral integro-differential inclusions with nonlocal initial conditions via fractional operators, Numer. Funct. Anal. Optim. 30 (2009), no. 3-4, 227-244.

[9] Y.-K. Chang, J. J. Nieto, and W. S. Li, On impulsive hyperbolic differential inclusions with nonlocal initial conditions, J. Optim. Theory Appl. 140 (2009), no. 3, 431-442.

[10] V. Daftardar-Gejji and S. Bhalekar, Boundary value problems for multi-term fractional differential equations, J. Math. Anal. Appl. 345 (2008), no. 2, 754-765.

[11] K. Deimling, Multivalued Differential Equations, Walter de Gruyter \& Co., Berlin, 1992.

[12] V. Gafiychuk, B. Datsko, and V. Meleshko, Mathematical modeling of time fractional reaction-diffusion systems, J. Comput. Appl. Math. 220 (2008), no. 1-2, 215-225.

[13] J. Henderson and A. Ouahab, Fractional functional differential inclusions with finite delay, Nonlinear Anal. 70 (2009), no. 5, 2091-2105. 
[14] S. Hu and N. Papageorgiou, Handbook of Multivalued Analysis. Vol. I, Kluwer Academic Publishers, Dordrecht, 1997.

[15] A. A. Kilbas, H. M. Srivastava, and J. J. Trujillo, Theory and Applications of Fractional Differential Equations, North-Holland Mathematics Studies, 204. Elsevier Science B.V., Amsterdam, 2006.

[16] S. Ladaci, J. L. Loiseau, and A. Charef, Fractional order adaptive high-gain controllers for a class of linear systems, Commun. Nonlinear Sci. Numer. Simul. 13 (2008), no. 4, $707-714$.

[17] A. Lasota and Z. Opial, An application of the Kakutani-Ky Fan theorem in the theory of ordinary differential equations, Bull. Acad. Polon. Sci. Ser. Sci. Math. Astronom. Phys. 13 (1965), 781-786.

[18] M. P. Lazarević, Finite time stability analysis of $\mathrm{PD}^{\alpha}$ fractional control of robotic timedelay systems, Mech. Res. Comm. 33 (2006), no. 2, 269-279.

[19] W. S. Li, Y. K. Chang, and J. J. Nieto, Solvability of impulsive neutral evolution differential inclusions with state-dependent delay, Math. Comput. Modelling 49 (2009), no. 9-10, 1920-1927.

[20] J. J. Nieto and R. Rodríguez-López, Euler polygonal method for metric dynamical systems, Inform. Sci. 177 (2007), no. 20, 4256-4270.

[21] A. Ouahab, Some results for fractional boundary value problem of differential inclusions, Nonlinear Anal. 69 (2008), no. 11, 3877-3896.

[22] I. Podlubny, Fractional Differential Equations, Academic Press, Inc., San Diego, CA, 1999.

[23] S. Z. Rida, H. M. El-Sherbiny, and A. A. M. Arafa, On the solution of the fractional nonlinear Schrodinger equation, Phys. Lett. A 372 (2008), no. 5, 553-558.

[24] S. G. Samko, A. A. Kilbas, and O. I. Marichev, Fractional Integrals and Derivatives, Gordon and Breach Science Publishers, Yverdon, 1993.

[25] G. V. Smirnov, Introduction to the Theory of Differential Inclusions, Graduate Studies in Mathematics, 41. American Mathematical Society, Providence, RI, 2002.

[26] S. Zhang, Existence of solution for a boundary value problem of fractional order, Acta Math. Sci. Ser. B Engl. Ed. 26 (2006), no. 2, 220-228.

Department of Mathematics

FACUlty of SCIENCE

King Abdulaziz University

P. O. Box 80203, Jeddah 21589, Saudi Arabia

E-mail address: bashir_qau@yahoo.com 